“C 2006 IEEE. Personal use of this material is permitted. Permission from IEEE must be obtained for all other uses, in any current or future media, including reprinting/republishing this material for advertising or promotional purposes, creating new collective works, for resale or redistribution to servers or lists, or reuse of any copyrighted component of this work in other works." 


\title{
Convergence Analysis for Extended Kalman Filter based SLAM
}

\author{
Shoudong Huang, Gamini Dissanayake \\ ARC Centre of Excellence for Autonomous Systems (CAS) \\ Faculty of Engineering \\ University of Technology, Sydney, Australia \\ \{sdhuang,gdissa\}@eng.uts.edu.au
}

\begin{abstract}
The main contribution of this paper is a theoretical analysis of the Extended Kalman Filter (EKF) based solution to the simultaneous localisation and mapping (SLAM) problem. The convergence properties for the general nonlinear two-dimensional SLAM are provided. The proofs clearly show that the robot orientation error has a significant effect on the limit and/or the lower bound of the uncertainty of the landmark location estimates. Furthermore, some insights to the performance of EKF SLAM and a theoretical analysis on the inconsistencies in EKF SLAM that have been recently observed are given.
\end{abstract}

\section{INTRODUCTION}

In the past decades, many different techniques have been developed to solve the SLAM problem (see for example, the recent papers [1] and the references therein). In traditional SLAM, Extended Kalman Filter (EKF) is used to estimate a state vector containing both the robot pose (including location and orientation) and the landmark locations (e.g. [2]).

There have been numerous implementations of the EKF SLAM algorithm. However, very few analytical results on the convergence and essential properties of the SLAM algorithm are available. Dissanayake et al. examined a simple linear version of the problem and provided convergence properties of SLAM and lower bound on the position uncertainty [2] [3] [4]. The results were extended to the multi-robots SLAM in [5], still in linear case. Recently, some results on analytical upper bound of SLAM position uncertainty are developed, assuming that an upper bound for the variance of the errors in the robot's orientation estimation can be determined a priori [6].

Almost all practical SLAM implementations have nonlinear process and observation models. The results due to [2] are intuitive and many early experiments and computer simulations appear to confirm that the properties of the linear solution extends to practical non-linear problems. However, in the past years, number of authors have demonstrated that the lower bound for the map accuracy presented in [2] is violated and the EKF SLAM produces inconsistent estimates due to errors introduced during the linearisation process [7] [8] [9] [10] [11]. While some explanation of this phenomena has been reported, mainly through monte-carlo simulations, a thorough theoretical analysis of the non-linear SLAM problem is not yet available.

In this paper, the convergence properties of EKF SLAM are analyzed for the general nonlinear case. The paper provides some key convergence properties and explicit formulas for the limits/lower bounds of the covariance matrices. Some insights and theoretical analysis of the EKF SLAM inconsistency are also given.

The paper is organized as follows. In Section II, a slightly different version of EKF SLAM algorithm is restated. In Section III, some key convergence properties are proved. The theoretical analysis of the EKF SLAM inconsistency are given in Section IV. Section V concludes the paper. Most of the complicated proofs in this paper are omitted due to the space limit. Interested readers please refer to a detailed version of this paper which is available online: http://services.eng.uts.edu.au/ sdhuang/publications.html

\section{EKF SLAM ALGORITHM RESTATEMENT}

The EKF SLAM algorithm is restated using slightly different notations which will help the statements and proofs of the results in this paper.

\section{A. State vector in $2 D$ EKF SLAM}

The state vector is denoted as:

$$
X=\left(\phi, X_{r}, X_{1}, \cdots, X_{n}\right) .
$$

Here $\phi$ is the robot orientation, $X_{r}=\left(x_{r}, y_{r}\right)$ is the robot position, $X_{1}=\left(x_{1}, y_{1}\right), \cdots, X_{n}=\left(x_{n}, y_{n}\right)$ are the positions of the $n$ landmarks. The reason why we separate the robot heading from the robot position is because $\phi$ plays a crucial role in the convergence and inconsistency analysis.

\section{B. Prediction}

1) Process Model: The robot process model considered in this paper is

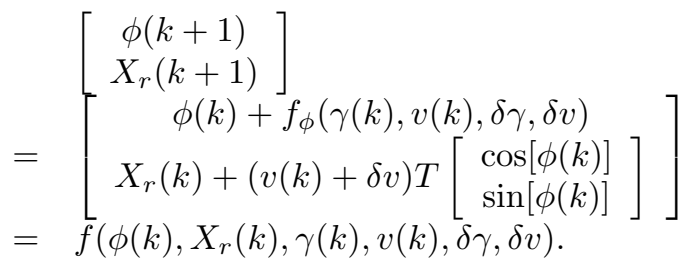

where $v, \gamma$ are the 'controls', $\delta v, \delta \gamma$ are zero-mean Gaussian noises on $v, \gamma . T$ is the time interval of one movement step. The explicit formula of function $f_{\phi}$ depends on the particular robot. The examples for this kind of process model include 
the direct disretization of the uni-cycle model (e.g. [10]) and a car-like vehicle model (e.g. [2]).

Model of landmarks (stationary) is

$$
X_{i}(k+1)=X_{i}(k), \quad i=1, \cdots, n .
$$

Thus, the process model of the whole state vector is

$$
X(k+1)=F(X(k), \gamma(k), v(k), \delta \gamma, \delta v)
$$

where $F$ is the function combining (2) and (3).

2) Prediction: Suppose at time $k$, the estimate of the state vector is

$$
\hat{X}(k)=\left(\hat{\phi}(k), \hat{X}_{r}(k), \hat{X}_{1}, \cdots, \hat{X}_{n}\right)
$$

and the covariance matrix of the estimation error is $P(k \mid k)$. Suppose the covariance of the control noises $(\delta \gamma, \delta v)$ is $\Sigma$, then the prediction step is

$$
\begin{aligned}
\hat{X}(k+1 \mid k) & =F(\hat{X}(k \mid k), \gamma(k), v(k), 0,0) \\
P(k+1 \mid k) & =\nabla F_{\phi X_{r} X} P(k \mid k) \nabla F_{\phi X_{r} X}^{T}+\nabla F_{\gamma v} \Sigma \nabla F_{\gamma v}^{T}
\end{aligned}
$$

where

$$
\nabla F_{\phi X_{r} X}=\left[\begin{array}{cc}
\nabla f_{\phi X_{r}} & 0 \\
0 & I
\end{array}\right], \nabla F_{\gamma v}=\left[\begin{array}{c}
\nabla f_{\gamma v} \\
0
\end{array}\right]
$$

and $\nabla f_{\phi X_{r}}$ and $\nabla f_{\gamma v}$ are Jacobians of $f$ in (2) with respect to the robot pose $\left(\phi, X_{r}\right)$ and the control noise $(\delta \gamma, \delta v)$, respectively, evaluated at the current estimation $\hat{X}(k \mid k)$.

For system (2),

$$
\nabla f_{\phi X_{r}}=\left[\begin{array}{ccc}
1 & 0 & 0 \\
-T v \sin [\hat{\phi}(k)] & 1 & 0 \\
T v \cos [\hat{\phi}(k)] & 0 & 1
\end{array}\right]
$$

The formula of $\nabla f_{\gamma v}$ depends on the detailed formula of function $f_{\phi}$ in (2).

\section{Update}

1) Measurement Model: At time $k+1$, the observation of $i$-th landmark is range $r_{i}$ and bearing $\theta_{i}$,

$$
\begin{aligned}
& r_{i}=\sqrt{\left(y_{i}-y_{r}(k+1)\right)^{2}+\left(x_{i}-x_{r}(k+1)\right)^{2}}+w_{r_{i}} \\
& \theta_{i}=\arctan \left(\frac{y_{i}-y_{r}(k+1)}{x_{i}-x_{r}(k+1)}\right)-\phi(k+1)+w_{\theta_{i}}
\end{aligned}
$$

where $w_{r_{i}}$ and $w_{\theta_{i}}$ are the noises on the measurements.

The observation model can be written in a general form as

$$
z_{i}(k+1)=\left[\begin{array}{c}
r_{i} \\
\theta_{i}
\end{array}\right]=H_{i}(X(k+1))+w_{r_{i} \theta_{i}}
$$

The noise $w_{r_{i} \theta_{i}}$ is assumed to be Gaussian with zero-mean and covariance matrix $R_{r_{i} \theta_{i}}$.
2) Update: To simplify the proofs of the results in this paper, we write the EKF covariance matrix update formula in the information form as

$$
\begin{aligned}
I(k+1 \mid k) & =P(k+1 \mid k)^{-1} \\
I(k+1 \mid k+1) & =I(k+1 \mid k)+I_{\text {new }} \\
P(k+1 \mid k+1) & =I(k+1 \mid k+1)^{-1}
\end{aligned}
$$

where

$$
I_{\text {new }}=\nabla H_{i}^{T} R_{r_{i} \theta_{i}}^{-1} \nabla H_{i}
$$

and $\nabla H_{i}$ is the Jacobian of function $H_{i}$ evaluated at the current estimation $\hat{X}(k+1 \mid k)$.

The update of the state vector estimation is

$$
\hat{X}(k+1 \mid k+1)=\hat{X}(k+1 \mid k)+W(k+1) \mu(k+1),
$$

where

$$
\begin{aligned}
\mu(k+1) & =z_{i}(k+1)-H_{i}(\hat{X}(k+1 \mid k)), \\
W(k+1) & =P(k+1 \mid k) \nabla H_{i}^{T} S^{-1}(k+1),
\end{aligned}
$$

and

$$
S(k+1)=R_{r_{i} \theta_{i}}+\nabla H_{i} P(k+1 \mid k) \nabla H_{i}^{T} .
$$

Remark 2.1: By (10),(11) and the matrix inversion lemma (e.g. [12]), we have

$$
\begin{aligned}
P(k+1 \mid k+1)= & P(k+1 \mid k)-P(k+1 \mid k) \nabla H_{i}^{T} \\
& \cdot\left(R_{r_{i} \theta_{i}}+\nabla H_{i} P(k+1 \mid k) \nabla H_{i}^{T}\right)^{-1} \\
& \cdot \nabla H_{i} P(k+1 \mid k),
\end{aligned}
$$

which is the typical EKF update formula.

The Jacobian of the measurement function $H_{i}$ is

$$
\nabla H_{i}=\left[\begin{array}{rrrrr}
0 & -\frac{d x}{r} & -\frac{d y}{r} & \frac{d x}{r} & \frac{d y}{r} \\
-1 & \frac{d y}{r^{2}} & -\frac{d x}{r^{2}} & -\frac{d y}{r^{2}} & \frac{d x}{r^{2}}
\end{array}\right]
$$

where

$$
\begin{aligned}
d x & =x_{i}-x_{r}(k+1) \\
d y & =y_{i}-y_{r}(k+1) \\
r & =\sqrt{d x^{2}+d y^{2}} .
\end{aligned}
$$

\section{Convergence Properties of EKF SLAM}

In this section, we prove some convergence results for $2 \mathrm{D}$ nonlinear EKF SLAM, these results are also true for the simple linear case.

\section{A. Monotonically decreasing property}

Theorem 3.1: The determinant of any submatrix of the map covariance matrix decreases monotonically as successive observations are made.

Theorem 3.1 is the same as Theorem 1 in [2] and can be proved in a similar way. The only difference is that the Jacobians instead of the state transition matrix and observation matrices will be used in the proof. The key point of the proof is "In the prediction step, the covariance matrix of the map is not changing; in the update step, the whole covariance matrix is non-increasing".

For 2D nonlinear EKF SLAM, there are many possible scenarios. In this paper, we only consider 2 basic scenarios: (1) robot keeps stationary and observe new landmarks many 
times, and (2) robot then moves but can only observe the same landmarks.

Suppose robot starts at point $\mathbf{A}$, the initial uncertainty of robot is

$$
P_{0}=\left[\begin{array}{cc}
p_{\phi} & \mathbf{p}_{x y \phi}^{T} \\
\mathbf{p}_{x y \phi} & P_{x y}
\end{array}\right]
$$

where $p_{\phi}$ is a scalar and $P_{x y}$ is a $2 \times 2$ matrix.

The initial information matrix is denoted as

$$
I_{0}=P_{0}^{-1}=\left[\begin{array}{cc}
i_{\phi} & \mathbf{b}^{T} \\
\mathbf{b} & I_{x y}
\end{array}\right] .
$$

\section{B. 2D EKF SLAM Scenario 1 - robot stationary}

Consider the scenario that the robot keeps stationary at point A and making observations $k \mapsto \infty$ times.

1) Observe one landmark: First assume robot can only observe one new landmark - landmark $m$. We denote the Jacobian in (16) evaluated at the true landmark position $\left(x_{m}, y_{m}\right)$ and the true robot position $\left(x_{A}, y_{A}\right)$ as ${ }^{1}$

$$
\nabla H_{A}=\left[\begin{array}{lll}
-\mathbf{e} & -A & A
\end{array}\right]
$$

where

$$
\mathbf{e}=\left[\begin{array}{l}
0 \\
1
\end{array}\right], \quad A=\left[\begin{array}{rr}
\frac{d x_{A}}{r_{A}} & \frac{d y_{A}}{r_{A}} \\
-\frac{d y_{A}}{r_{A}^{2}} & \frac{d x_{A}}{r_{A}^{2}}
\end{array}\right]
$$

with

$$
\begin{aligned}
d x_{A} & =x_{m}-x_{A}, \\
d y_{A} & =y_{m}-y_{A}, \\
r_{A} & =\sqrt{d x_{A}^{2}+d y_{A}^{2}} .
\end{aligned}
$$

For convenient, we further denote

$$
H_{A}=\left[\begin{array}{ll}
\mathbf{e} & A
\end{array}\right] \text {. }
$$

Theorem 3.2: If robot keeps stationary and observe a new landmark many times, the robot uncertainty keeps unchanged. In the limit, the uncertainty of the landmark estimation is

$$
P_{A_{m}}^{\infty}=A^{-1} H_{A} P_{0} H_{A}^{T} A^{-T},
$$

where $P_{0}$ is the initial robot uncertainty given in (18), $A$ is defined by (21), and $H_{A}$ is defined in (23). In the special case when the initial uncertainty of robot orientation $p_{\phi}$ is 0 , the limit $P_{A_{m}}^{\infty}$ is equal to the initial robot position uncertainty $P_{x y}$ in (18).

Remark 3.3: Theorem 3.2 is the nonlinear version of Theorem 3 in [2]. Moreover, we state clearly here that the robot orientation estimation error has significant effect on the limit of the landmark uncertainty. This does make sense because: "when the robot position is exactly known but its orientation is uncertain, even if there is a perfect knowledge about the relative location between the landmark and the robot, it is still impossible to tell where the true landmark location is".

\footnotetext{
${ }^{1}$ For the theoretical convergence results, we always evaluate the Jacobians at the true values, in the real SLAM application, the Jacobians have to be evaluated at the estimated value and this may cause inconsistency, see Section IV.
}

Figures 1(a) and 1(b) show the different landmark uncertainties due to different robot orientation estimation errors.

Proof of Theorem 3.2: We denote the observation noise covariance matrix as $R_{A}$. The information gain from one observation is:

$$
I_{\text {new }}=\nabla H_{A}^{T} R_{A}^{-1} \nabla H_{A} .
$$

The total information after the $k$ observations is

$$
\begin{aligned}
= & {\left[\begin{array}{cc}
I_{A_{\text {end }}}^{k} & 0 \\
0 & 0
\end{array}\right]+k\left[\begin{array}{c}
-H_{A}^{T} \\
A^{T}
\end{array}\right] R_{A}^{-1}\left[\begin{array}{ll}
-H_{A} & A
\end{array}\right] } \\
= & {\left[\begin{array}{cc}
I_{0}+k H_{A}^{T} R_{A}^{-1} H_{A} & -k H_{A}^{T} R_{A}^{-1} A \\
-k A^{T} R_{A}^{-1} H_{A} & k A^{T} R_{A}^{-1} A
\end{array}\right] . }
\end{aligned}
$$

By the matrix inversion lemma (e.g. [12])

$$
\begin{aligned}
P_{A_{\text {end }}}^{k} & =\left(I_{A_{\text {end }}}^{k}\right)^{-1} \\
& =\left[\begin{array}{cc}
I_{0}^{-1} & I_{0}^{-1} H_{A}^{T} A^{-T} \\
A^{-1} H_{A} I_{0}^{-1} & P_{A_{m}}^{k}
\end{array}\right] \\
& =\left[\begin{array}{cc}
P_{0} & P_{0} H_{A}^{T} A^{-T} \\
A^{-1} H_{A} P_{0} & P_{A_{m}}^{k}
\end{array}\right]
\end{aligned}
$$

where

$$
P_{A_{m}}^{k}=A^{-1} H_{A} P_{0} H_{A}^{T} A^{-T}+\frac{A^{-1} R_{A} A^{-T}}{k} .
$$

When $k \rightarrow \infty$, the second item goes to 0 , so we have (24). The limit can be computed further as

$$
\begin{aligned}
P_{A_{m}}^{\infty}= & A^{-1}\left[\begin{array}{ll}
\mathbf{e} & A
\end{array}\right]\left[\begin{array}{cc}
p_{\phi} & \mathbf{p}_{x y \phi}^{T} \\
\mathbf{p}_{x y \phi} & P_{x y}
\end{array}\right]\left[\begin{array}{c}
\mathbf{e}^{T} \\
A^{T}
\end{array}\right] A^{-T} \\
= & P_{x y}+A^{-1} \mathbf{e} p_{\phi} \mathbf{e}^{T} A^{-T} \\
& +A^{-1} \mathbf{e p}_{x y \phi}^{T}+\mathbf{p}_{x y \phi} \mathbf{e}^{T} A^{-T}
\end{aligned}
$$

When $p_{\phi} \rightarrow 0$ (then $\mathbf{p}_{x y \phi} \rightarrow 0$ because $P_{0}$ is positive definite), the limit $P_{A_{m}}^{\infty} \rightarrow P_{x y}$. The proof is completed.

2) Observe two landmarks: Suppose robot can observe two new landmarks (landmark $m$ and landmark $\bar{m}$ ) at point $\mathbf{A}$, then the dimension of the observation function in (9) is 4 (two ranges and two bearings), we denote the Jacobian as:

$$
\nabla \hat{H}_{A}=\left[\begin{array}{ccc}
-H_{A} & A & 0 \\
-H_{\bar{A}} & 0 & \bar{A}
\end{array}\right]=\left[\begin{array}{cccc}
-\mathbf{e} & -A & A & 0 \\
-\mathbf{e} & -\bar{A} & 0 & \bar{A}
\end{array}\right] .
$$

Theorem 3.4: If robot keeps stationary and observes two new landmarks many times, the robot uncertainty keeps unchanged. In the limit, the uncertainty of the landmarks is

$$
P_{m \bar{m}}^{\infty}=\left[\begin{array}{cc}
P_{A m}^{\infty} & P_{A \bar{A}} \\
P_{A \bar{A}}^{T} & P_{\bar{A}_{\bar{m}}}^{\infty}
\end{array}\right]
$$

where

$$
\begin{aligned}
P_{A_{m}}^{\infty} & =A^{-1} H_{A} P_{0} H_{A}^{T} A^{-T}, \\
P_{A \bar{A}} & =A^{-1} H_{A} P_{0} H_{\bar{A}}^{T} \bar{A}^{-T}, \\
P_{\bar{A}_{\bar{m}}}^{\infty} & =\bar{A}^{-1} H_{\bar{A}} P_{0} H_{\bar{A}}^{T} \bar{A}^{-T} .
\end{aligned}
$$

In the special case when the initial uncertainty of robot orientation is 0 , the limit $P_{m \bar{m}}^{\infty}=\left[\begin{array}{cc}P_{x y} & P_{x y} \\ P_{x y} & P_{x y}\end{array}\right]$. 


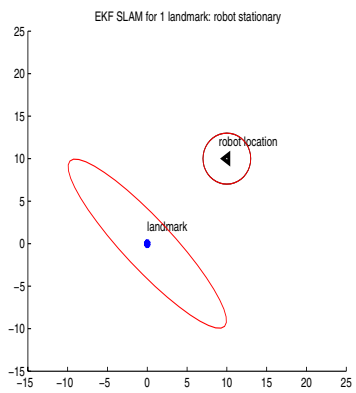

(a) Initial robot orientation error is (b) Initial robot orientation error is large

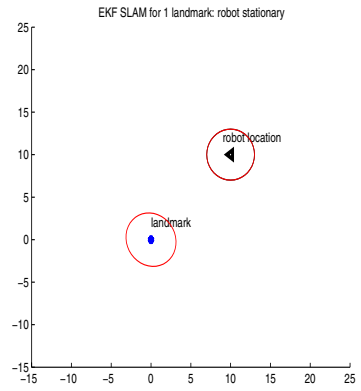

small

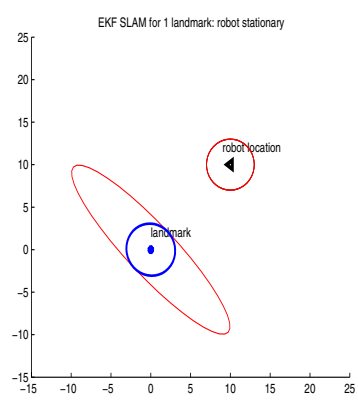

(c) Inconsistency of EKF SLAM

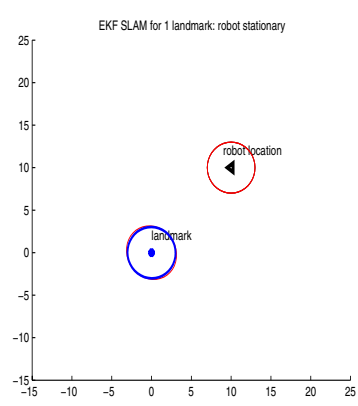

(d) Inconsistency can be neglected

Fig. 1. The limits of landmark uncertainty when robot keeps stationary and observe the landmark $k \rightarrow \infty$ times (see Theorem 3.2 and Theorem 4.1 ): In Figure 1(a), the variance of the robot orientation estimation error is $p_{\phi}=0.1$, the initial uncertainty of robot is $P_{0}=\operatorname{diag}(0.1,1,1)$. In Figure $1(\mathrm{~b})$, the variance of the robot orientation estimation error is $p_{\phi}=0.001$, the initial robot uncertainty is $P_{0}=\operatorname{diag}(0.001,1,1)$. Because the robot orientation error is very small, in the limit, the uncertainty of the landmark is very close to the initial uncertainty of the robot position. For these two figures, the Jacobins are evaluated at the true robot and landmark positions. These two figures show that the initial robot orientation error has a significant effect on the landmark estimation accuracy. In Figure 1(c), the initial uncertainty of robot is the same as that in Figure 1(a), the blue ellipse is the limit of the landmark uncertainty when the Jacobian is evaluated at the updated state estimation at each update step. It can be seen that the uncertainty of the landmark reduces to the same size as the initial robot position estimation error, this is the inconsistency of EKF SLAM algorithm. In Figure 1(d), the initial uncertainty of robot is the same as that in Figure 1(b), it can be seen that the inconsistency can be neglected in this case (the blue ellipse almost coincides with the red one).

Remark 3.5: Because $A^{-1} H_{A} \neq \bar{A}^{-1} H_{\bar{A}}, P_{A_{m}}^{\infty} \neq P_{\bar{A}_{\bar{m}}}^{\infty}$ when $p_{\phi} \neq 0$. This means the limits of the uncertainties of the two landmarks are different when the robot orientation error is not zero. This is different from the linear results proved in [2], where the uncertainties of all the landmarks are the same (Theorem 2 in [2]). This result is due to the nonlinearity of the observation function, the Jacobians are different when evaluated at locations of different landmarks. Figure 2(a) shows the difference of the uncertainties of the two landmarks.

\section{2D EKF SLAM Scenario 2 - robot moves}

Consider the scenario that the robot first keeps stationary at point $\mathbf{A}$ and observing landmark $m$ many times. Then the robot moves to another observation point $\mathbf{B}$, and observes the same landmark many times.

We use $\nabla f_{\phi X_{r}}^{A}, \nabla f_{\gamma v}^{A}$ to denote the Jacobians of $f$ evaluated at the point $\mathbf{A}$ and the true landmark location. We denote the Jacobian in (16) evaluated at point $\mathbf{B}$ and the true location of landmark $m$ as

$$
\nabla H_{B}=\left[\begin{array}{lll}
-\mathbf{e} & -B & B
\end{array}\right]
$$

and denote

$$
H_{B}=\left[\begin{array}{ll}
\mathbf{e} & B
\end{array}\right] .
$$

Theorem 3.6: If robot first keeps stationary at point A and observes one new landmark $k \rightarrow \infty$ times, then it moves to point $B$ and observes the same landmark $l \rightarrow \infty$ times, the limit of the covariance matrix satisfies

$$
P_{B_{\text {end }}}^{\infty} \geq\left[\begin{array}{cc}
\nabla f_{\phi X_{r}}^{A} P_{0}\left(\nabla f_{\phi X_{r}}^{A}\right)^{T} & \nabla f_{\phi X_{r}}^{A} P_{0} H_{A}^{T} A^{-T} \\
A^{-1} H_{A} P_{0}\left(\nabla f_{\phi X_{r}}^{A}\right)^{T} & A^{-1} H_{A} P_{0} H_{A}^{T} A^{-T}
\end{array}\right]
$$

The righthand side matrix is actually the covariance matrix when robot first reaches point $B$ assuming there is no control noise moving from $A$ to $B(\Sigma=0$ in (5)). Furthermore, the inequality in (35) becomes equality when either (i) $\Sigma=0$, or (ii) $\Sigma \neq 0$ but the matrix $H_{B} \nabla f_{\gamma v}^{A}$ is invertible (which is true in most of the cases).

Remark 3.7: Theorem 3.6 shows that the only effect of the observations made at point $\mathbf{B}$ is to reduce the robot uncertainty generated from the process noise. The observations made at point $\mathbf{B}$ can not reduce the uncertainty of the landmark further if robot had already observed the landmark many times at point A. Theorem 3.6 can be extended to the case when it takes a few steps to move from $\mathbf{A}$ to $\mathbf{B}$, for example, $A \rightarrow$ $B_{1} \rightarrow B_{2} \rightarrow \ldots \rightarrow B_{n} \rightarrow B$. In this case, the limit of the covariance matrix satisfies

$$
P_{B_{\text {end }}}^{\infty} \geq\left[\begin{array}{cc}
F_{A B} P_{0} F_{A B}^{T} & F_{A B} P_{0} H_{A}^{T} A^{-T} \\
A^{-1} H_{A} P_{0} F_{A B}^{T} & A^{-1} H_{A} P_{0} H_{A}^{T} A^{-T}
\end{array}\right] .
$$

where

$$
F_{A B}=\nabla f_{\phi X_{r}}^{B_{n}} \cdots \nabla f_{\phi X_{r}}^{B_{1}} \nabla f_{\phi X_{r}}^{A} .
$$

Figures 3(a), 3(b), 3(c), and 3(d) illustrate the results in Theorem 3.6 and Remark 3.7.

\section{INCONSISTENCY OF EKF SLAM}

Recently, it is reported that EKF SLAM may produce inconsistent (overconfident) estimation [7] [8] [9] [10] [11]. However, most of the claims are based on Monte Carlo simulations.

The limits/lower bounds of the covariance matrix proved in the last section can be used for the check of inconsistency. For example, the results in the last section shows that the uncertainty of a new landmark first observed at point $\mathbf{A}$ can not be smaller than $A^{-1} H_{A} P_{0} H_{A}^{T} A^{-T}$ if robot can not observe any old landmarks. 


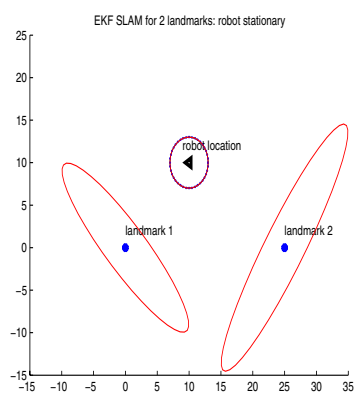

(a) Initial robot orientation error is large

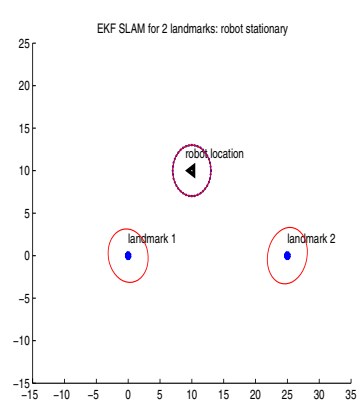

(b) Init
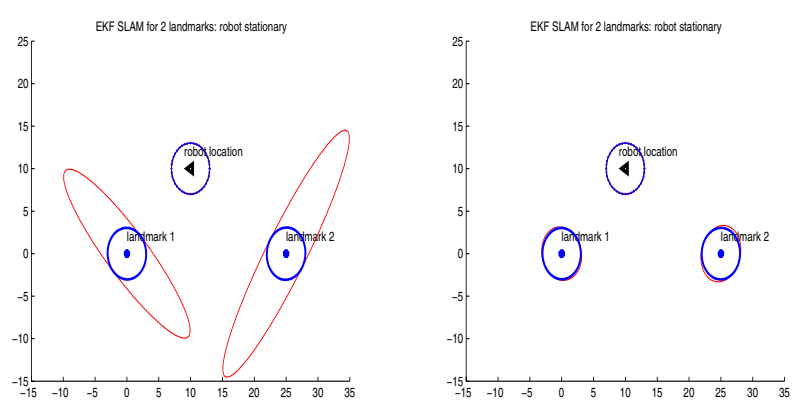

is (c) Inconsistency of EKF SLAM for two landmarks

Fig. 2. The limits of two landmark uncertainties when robot keeps stationary and make observation $k \rightarrow \infty$ times: Figure 2 (a) shows that the final uncertainties of the two landmarks are different; Figure 2(b) shows that the difference is very small when the initial robot orientation uncertainty $p_{\phi}$ is small; Figure 2(c) shows the inconsistency of EKF SLAM, Figure 2(d) shows the inconsistency can be neglected when $p_{\phi}$ is small (see Theorem 3.4, Remark 3.5, Theorem 4.1 and the caption of Figure 1 for more explanations)

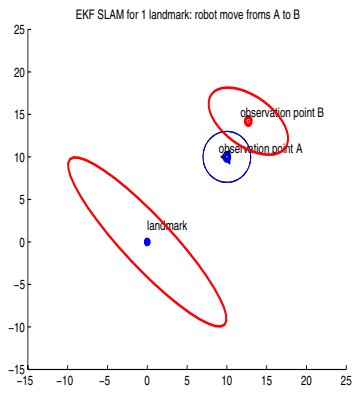

(a) no control noise

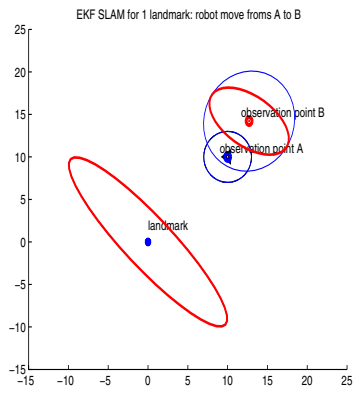

(b) with control noise
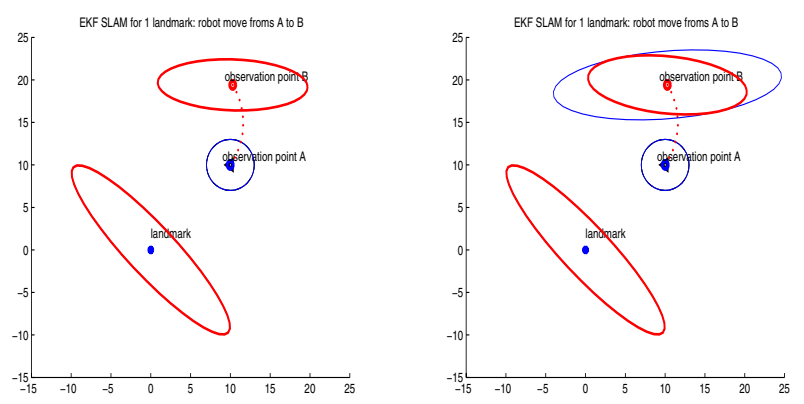

(c) move 10 steps with no control (d) move 10 steps with control noise noise

Fig. 3. The limits of robot and landmark uncertainties when robot first keeps stationary at point $\mathbf{A}$ and makes observation $k \rightarrow \infty$ times and then moves to $\mathbf{B}$ and observes the same landmark $l \rightarrow \infty$ times (see Theorem 3.6 and Remark 3.7): The initial robot uncertainty is the same as that in Figure 1(a), Figure 3(a) shows the case when there is no control noise, the uncertainty of robot and landmarks keep unchanged after the observations at point $\mathbf{B}$, Figure 3(b) shows the case when there is control noise, the landmark uncertainty can not be improved by the observation at point $\mathbf{B}$, while the uncertainty of robot can be reduced to the same level as the case when there is no control noise, Figures 3(c) and 3(d) shows the similar results when it takes 10 steps to move from A to $\mathbf{B}$.

\section{A. Why inconsistency can occur in nonlinear case?}

In all the theoretical convergence properties proved in the last section, it is assumed that the Jacobians are evaluated at the true observation point and the true landmark positions. In a real SLAM, the true locations of robot and landmarks are not available, and the Jacobians have to be evaluated at the estimated values. It is well known that this kind of linearization error can cause incorrect estimation. However, incorrect estimation may be either too optimistic (estimated uncertainty smaller than true uncertainty) or too pessimistic (estimated uncertainty larger than true uncertainty). But it is only reported that EKF SLAM may cause inconsistency (optimism), no one have claimed EKF SLAM may be pessimism. Why?

\section{B. An intuitive explanation}

In SLAM, the observation is always the relative location between robot and landmarks, and we want to estimate the absolute robot and landmark locations.

Suppose $x, y$ are two numbers and the only thing we can measure is $x-y$. Suppose we have obtained two (noisy) measurements:

$$
\begin{aligned}
& x-y=99.8 \\
& x-y=100.1
\end{aligned}
$$

According to these measurements we may be able to say " $x-y$ is around 100 " but we can not say anything about the value of $x$ and/or $y$. However, with "linearization error", we may get something like

$$
\begin{aligned}
& 1.01 x-0.98 y=99.8 \\
& 0.99 x-1.02 y=100.1
\end{aligned}
$$

By the above two equations, the absolute values of $x$ and $y$ can be calculated. Obviously, the result is too optimistic (inconsistent).

In SLAM, suppose robot keeps stationary at point $\mathbf{A}$ and make two observations at a new landmark $m$. In EKF SLAM, we always use the observation innovation $(\mu(k+1)$ in (13)) to update our previous estimation $\hat{X}$, through linearization, the innovation is the information about

$H(X)-H(\hat{X})=\nabla H(X-\hat{X})=\mathbf{e} \Delta \phi+A \Delta X_{r}-A \Delta X_{m}$. 
When the Jacobian $\nabla H$ are evaluated at two different estimations, the two innovations $\mu_{1}, \mu_{2}$ give

$$
\begin{aligned}
& \mathbf{e} \Delta \phi+\tilde{A}_{1} \Delta X_{r}-\tilde{A}_{1} \Delta X_{m}=\mu_{1}, \\
& \mathbf{e} \Delta \phi+\tilde{A}_{2} \Delta X_{r}-\tilde{A}_{2} \Delta X_{m}=\mu_{2},
\end{aligned}
$$

If $\tilde{A}_{1} \neq \tilde{A}_{2}$, then we may be able to solve the absolute value of $\Delta \phi$. This means we can improve out knowledge on the robot orientation $\phi$. It is obvious that the robot orientation error can not be reduced by observing a single new landmark. So this is wrong (inconsistent).

In the next subsection, we will provide some theoretical results of the inconsistency for the scenario when robot keeps stationary.

\section{Inconsistency when robot keeps stationary}

Consider the scenario that the robot keeps stationary at point A and observing a new landmark $k$ times. The estimation is updated after each observation and the Jacobian is evaluated at the updated estimation at each time step. Denote the different Jacobians as

$$
\nabla H_{\tilde{A}_{j}}=\left[\begin{array}{lll}
-\mathbf{e} & -\tilde{A}_{j} & \tilde{A}_{j}
\end{array}\right], \quad 1 \leq j \leq k .
$$

We use $R_{\tilde{A}_{j}}$ to denote the observation noise covariance matrix of the $j$-th observation, and define

$$
\begin{aligned}
w(k, A)= & \sum_{j=1}^{k} \mathbf{e}^{T} R_{\tilde{A}_{j}}^{-1} \mathbf{e}-\mathbf{e}^{T}\left(\sum_{j=1}^{k} R_{\tilde{A}_{j}}^{-1} \tilde{A}_{j}\right) \\
& \cdot\left(\sum_{j=1}^{k} \tilde{A}_{j}^{T} R_{\tilde{A}_{j}}^{-1} \tilde{A}_{j}\right)^{-1}\left(\sum_{j=1}^{k} \tilde{A}_{j}^{T} R_{\tilde{A}_{j}}^{-1}\right) \mathbf{e} .
\end{aligned}
$$

Suppose the initial robot uncertainty is $P_{0}$ given by (18).

Theorem 4.1: In EKF SLAM, if the robot keeps stationary at point $A$ and observing a new landmark $k$ times, the inconsistency may occur due to the fact that Jacobians are evaluated at different estimation values. The level of inconsistency is decided by the initial robot uncertainty $P_{0}$ and the $w(k, A)$ defined in (42). When $k \rightarrow \infty$, the inconsistency may cause the robot orientation error $p_{\phi}$ to be reduced to zero.

Figures 1(c), 1(d), 2(c), and 2(d) illustrate the results in Theorem 4.1.

\section{CONCLUSiON}

In this paper, it is shown that most of the convergence properties of the EKF based SLAM algorithm, proved by Dissanayake et al. [2] can be generalized to be applicable to practical nonlinear 2D SLAM problems. Explicit formulas for the limits/lower bounds of the covariance matrices are provided for several scenarios. It is clearly shown that robot orientation error plays a key role in the EKF SLAM convergence and consistency analysis. Some insights on EKF SLAM inconsistency are also given. It is shown that when the robot orientation error is large, the inconsistency can become a fatal problem. This confirms the empirical findings by Tim Bailey [11].

Our ongoing research include the investigation of the limits/lower bounds of the covariance matrices and analysis of the inconsistency issue for more complicated scenarios. The next step of the research is to develop robust implementations of EKF SLAM that maximise the convergence rate and minimise the effect of factors that lead to filter inconsistency.

\section{ACKNOWLEDGMENT}

This work is supported by the ARC Centre of Excellence programme, funded by the Australian Research Council (ARC) and the New South Wales State Government. The authors would also like to thank Dr. Tim Bailey for helpful discussions.

\section{REFERENCES}

[1] U. Frese, P. Larsson, T. Duckett, "A multilevel relaxation algorithm for simultaneous localization and mapping," IEEE Transactions on Robotics, vol. 21 (2), pp. 196- 207, 2005.

[2] G. Dissanayake, P. Newman, S. Clark, H. Durrant-Whyte, and M. Csorba, "A solution to the simultaneous localization and map building (SLAM) problem," IEEE Trans. on Robotics and Automation, vol. 17, pp. 229-241, 2001.

[3] P. W. Gibbens, G. Dissanayake, and H. Durrant-Whyte, "A closed form solution to the single degree of freedom simultaneous localization and map building (SLAM) problem," Proceeding of the 39th IEEE Conferences on Decision and Control, Sydney, Australia, December 2000, pp. 191-196.

[4] P. M. Newman, On the Structure and Solution of the Simultaneous Localization and Map Building Problem, PhD thesis, Australian Centre of Field Robotics, University of Sydney, Sydney, 2000. Available online http://www.robots.ox.ac.uk/ pnewman/

[5] J. W. Fenwick, P. M. Newman and J. J. Leonard, "Cooperative concurrent mapping and localization", Proceeding of the IEEE Conference on Robotics and Automation, Washington D.C. May 2002, pp. 1810-1817.

[6] A. I. Mourikis, S. I. Roumeliotis, Analysis of Positioning Uncertainty in Simultaneous Localization and Mapping (SLAM), In Proceedings of the 2004 IEEE/RSJ International Conference on Intelligent Robots and Systems, September 28 - October 2, 2004, Sendai, Japan, pp. 13-20.

[7] S. J. Julier and J. K. Uhlmann, "A counter example for the theory of simultaneous localization and map building", Proceeding of the IEEE Conference on Robotics and Automation, Seoul, Korea, May 21-26, pp. 4238-4243, 2001.

[8] J.A. Castellanos, J. Neira and J.D. Tardos. Limits to the consistency of EKF-based SLAM. 5th IFAC Symp. on Intelligent Autonomous Vehicles, IAV'04, Lisbon, Portugal, July 2004. available online http://webdiis.unizar.es/\%7Ejdtardos/publications.html

[9] U. Frese. A Discussion of Simultaneous Localization and Mapping. Autonomous Robots (to appear). available online http://www.informatik.uni-bremen.de/ũfrese/publications_e.html

[10] A. Martinell, N.Tomatis, and R. Siegwart, Some Results on SLAM and the Closing the Loop Problem. In Proceedings of the IEEE/RSJ Intenational Conference on Intelligent Robots and Systems, IROS, Edmonton, Canada, 2005, 334-339.

[11] Tim Bailey, Juan Nieto, Jose Guivant and Eduardo Nebot, Consistency of the EKF-SLAM Algorithm, submitted to ISRR 2005, available online http://www.acfr.usyd.edu.au/

[12] F. Zhang, Matrix Theory: Basic Results and Techniques, SpringerVerlag. 1999. 